\title{
Predictive Voltage Control of the Direct Matrix Converter applied to Grid Current Injection
}

\author{
S. Toledo, M. Rivera, T. Dragicevic, D. Caballero, P. Wheeler and R. Gregor
}

\begin{abstract}
Predictive control applied to direct matrix converter provide a feasible option to control the output voltage of a generation system with good performance. In this work a predictive voltage control strategy is proposed as the inner loop control of an outer PR current control strategy to inject controlled power to the main distribution grid from an AC generation system. The proposal shows good performance in terms of THD and desired current traking.
\end{abstract}

keywords-predictive voltage control, distributed generation, matrix converter, current control.

\section{INTRODUCCIÓN}

La creciente demanda de energía a nivel mundial y los conocidos efectos nocivos de los combustibles fósiles hacen necesario el desarrollar nuevos esquemas de generación de energía a partir de fuentes renovables y amigables con el medio ambiente [1]-[3]. En los últimos años, el control predictivo basado en modelo (MPC) ha sido implementado de manera exitosa en varias aplicaciones de electrónica de potencia [4], [5], como el control de par mecánico y flujo electromagnético [6], [7], control de corriente y potencia [8], [9], así como en fuentes de suministro ininterrumpido (UPS) [10], [11]. Así también, el MPC ha surgido como una opción plausible para la conmutación controlada de los interruptores en convertidores de potencia para aplicaciones en sistemas de generación distribuida [12]-[14]. El objetivo de estos sistemas es inyectar corrientes controladas de calidad al sistema de distribución principal y por ende la potencia activa y reactiva.

Uno de los principales elementos para implementar una estrategia de control MPC es un modelo preciso del sistema de manera a tener una predicción adecuada. El modelo depende directamente de la aplicación que se desea dar al sistema [15].

En el caso de los convertidores de potencia, estos están compuestos por semiconductores que operan en sólo dos estados: corte y saturación. Por lo tanto, siempre existe un número finito de posibles combinaciones de los estados de conmutación en cualquier convertidor de potencia. Esta característica simplifica en gran medida la aplicación del MPC, ya

Los autores expresan su gratitud al Consejo Nacional de Ciencia y Tecnología del Paraguay (CONACYT), por el apoyo y financiamiento a través del Proyecto PINV15-0584, al CONICYT de Chile a través del Proyecto FONDECYT Regular 1160690, el Proyecto MEC 80150056.

S. Toledo y M. Rivera, investigadores del Laboratorio de Conversión de Energías y Electrónica de Potencia, Facultad de Ingeniería, Universidad de Talca, Curicó, Chile, e-mail: (ver en http://marcorivera.cl).

T. Dragicevic es investigador adscripto al Departamento de Tecnologías Energéticas de la Aalborg University, 9220 Aalborg East, Denmark (e-mail: tdr@et.aau.dk)

D. Caballero y R. Gregor, investigadores del Laboratorio de Sistemas de Potencia y Control, Facultad de Ingeniería UNA, Luque, CP2060, Paraguay, e-mail: (ver en http://www.dspyc.com.py/equipo.html). que, en lugar de tener que perder tiempo en un algoritmo de optimización continua, es posible realizar la evaluación directa de todos los estados de conmutación posibles y seleccionar la mejor combinación a utilizar de acuerdo a los objetivos de control propuestos.

Los objetivos de control en el MPC son representados mediante una función de costo, la cual evalúa los errores entre las referencias y los valores reales de las variables de interés. Además, es posible agregar algunos objetivos de control adicionales como control de temperatura y minimización de las pérdidas por conmutación y conducción [16], tensiones de modo común [17], entre otros. Así también, operaciones no lineales, como restricciones y limitaciones pueden ser fácilmente agregadas, aumentando en gran medida la flexibilidad y riqueza de la estrategia de control. Es posible separar este principio de operación en tres pasos (i) cálculo de la variables predichas utilizando el modelo discreto, (ii) evaluación de la función de costo para cada uno de los estados válidos del convertidor, y (iii) selección y aplicación del estado de conmutación óptimo. Estas etapas están presentes en todos los esquemas de MPC, ya que es posible modificar cada una independientemente para ajustar el esquema de control a cualquier configuración.

En este trabajo se presenta una propuesta de control de corriente inyectada a la red de distribución principal con base en un lazo de control interno en el cual se implementa un control predictivo de tensión de salida para un convertidor matricial directo y un lazo de control externo proporcional derivativo $(\mathrm{PR})$ con una señal de prealimentación para general la tensión de referencia del control MPC de tensión.

\section{ESQUEMA GENERAL DE SISTEMAS DE GENERACIÓN DISTRIBUIDA INTERCONECTADOS A LA RED}

$\mathrm{El}$ esquema general de un sistema interconectado a la red se muestra en la Fig. 1. Esta topología permite inyectar corriente eléctrica a la red principal en base al control de tensión en el capacitor del filtro de salida. El bloque PLL (Lazo de amarre de fase) mide la fase de la tensión de la red de manera a generar una tensión sincronizada con la misma a la salida del convertidor. Este proceso se lleva a cabo para interconectar ambos sistemas y poder inyectar energía. De esta manera se debe diseñar un esquema de control interior de tensión y un lazo de control de corriente exterior que proporcione la tensión de referencia al lazo interno para generar la corriente deseada.

\section{A. Convertidor Matricial Directo. Principios Básicos}

Para introducir los principios básicos referentes a los convertidores matriciales, se considera el sistema de conversión 


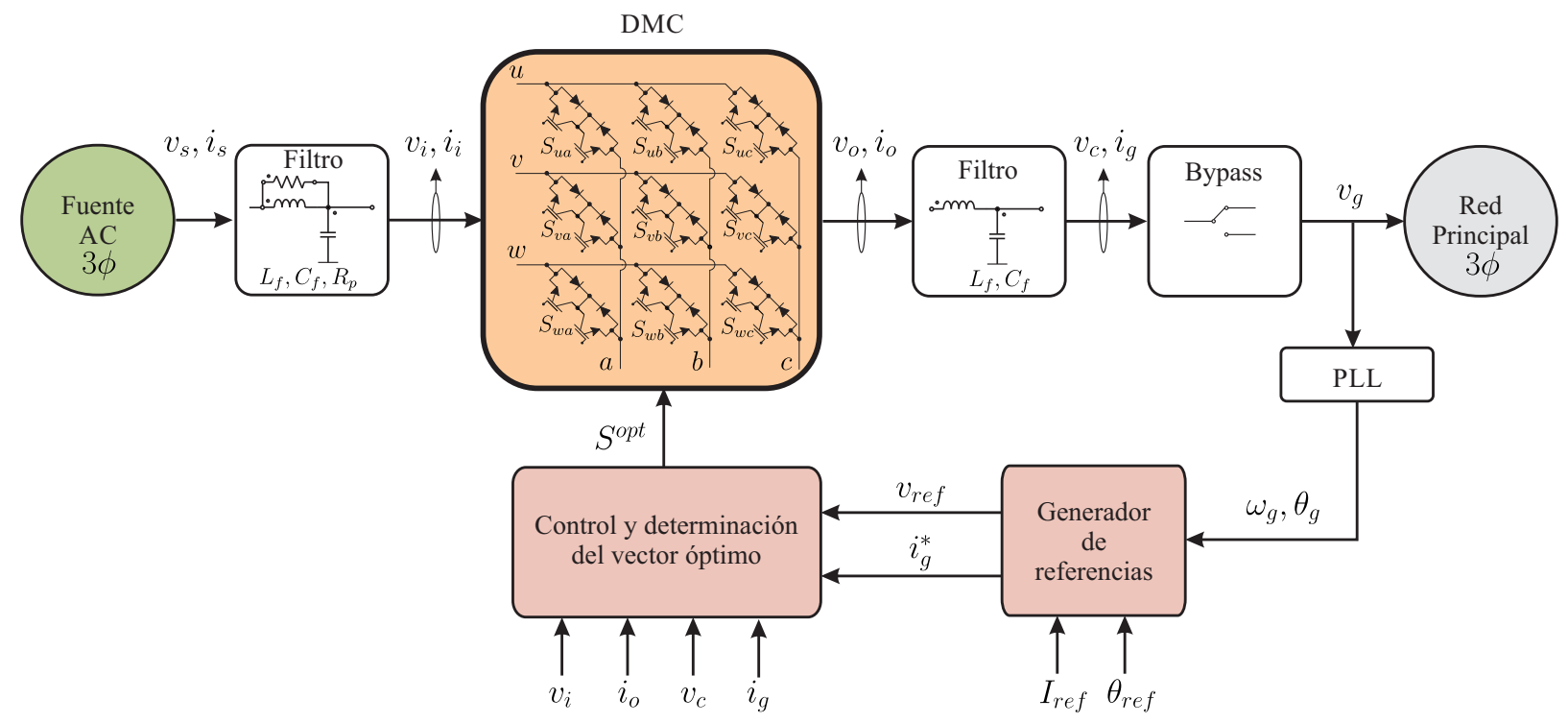

Fig. 1. Esquema General del Sistema Conectado a la red.

AC-AC trifásico de la Fig. 1.

La función de conmutación para un interruptor simple se define como:

$$
S_{i j}=\left\{\begin{array}{ll}
0, & \text { interruptor } S_{i j} \text { desactivado } \\
1, & \text { interruptor } S_{i j} \text { activado }
\end{array},\right.
$$

donde $i \in\{u, v, w\}$ indica la entrada correspondiente, $k \in\{a, b, c\}$ se refiere a la salida correspondiente. Considerando que las entradas nunca deben conectarse en corto circuito y que las corrientes nunca deben interrumpirse abruptamente, se expresan las restricciones de la siguiente forma:

$$
S_{u i}+S_{v i}+S_{w i}=1, \quad \forall i \in\{a, b, c\} .
$$

Bajo estas restricciones, el MC trifásico presenta 27 estados de conmutación permitidos, de entre los $512\left(2^{9}\right)$ posibles. En la Tabla I se presentan todos los estados válidos en función a la restricción de la ecuación (2).

Si la carga y la fuente son referenciadas respecto al punto neutro $(N)$, entonces es posible describir la relación entre las entradas y las salidas de tensión y de corriente como sigue:

$$
\left[\begin{array}{c}
v_{a}(t) \\
v_{b}(t) \\
v_{c}(t)
\end{array}\right]=\left[\begin{array}{lll}
S_{u a}(t) & S_{v a}(t) & S_{w a}(t) \\
S_{u b}(t) & S_{v b}(t) & S_{w b}(t) \\
S_{u c}(t) & S_{v c}(t) & S_{w c}(t)
\end{array}\right]\left[\begin{array}{c}
v_{u}(t) \\
v_{v}(t) \\
v_{w}(t)
\end{array}\right],
$$

$$
\left[\begin{array}{c}
i_{u}(t) \\
i_{v}(t) \\
i_{w}(t)
\end{array}\right]=\left[\begin{array}{ccc}
S_{u a}(t) & S_{u b}(t) & S_{u c}(t) \\
S_{v a}(t) & S_{v b}(t) & S_{v c}(t) \\
S_{w a}(t) & S_{w b}(t) & S_{w c}(t)
\end{array}\right]\left[\begin{array}{c}
i_{o a}(t) \\
i_{o b}(t) \\
i_{o c}(t)
\end{array}\right]
$$

Una vez definido el modelo del convertidor de potencia, se procede a diseñar el sistema de control.

Para determinar las tensiones efectivas aplicadas a cada fase (i.e., desde $a, b, y c$ al punto $n$ ), la tensión en modo común $v_{n N}$ debe ser sustraída de la ecuación (3). Es posible determinar
TABLA I

ESTADOS VÁLIDOS PARA EL CONVERTIDOR MATRICIAL.

\begin{tabular}{cccccccccc}
\hline & $S_{u a}$ & $S_{v a}$ & $S_{w a}$ & $S_{u b}$ & $S_{v b}$ & $S_{v c}$ & $S_{w a}$ & $S_{w b}$ & $S_{w c}$ \\
\hline 1 & 1 & 0 & 0 & 1 & 0 & 0 & 1 & 0 & 0 \\
2 & 0 & 1 & 0 & 1 & 0 & 0 & 1 & 0 & 0 \\
3 & 0 & 0 & 1 & 1 & 0 & 0 & 1 & 0 & 0 \\
4 & 1 & 0 & 0 & 0 & 1 & 0 & 1 & 0 & 0 \\
5 & 0 & 1 & 0 & 0 & 1 & 0 & 1 & 0 & 0 \\
6 & 0 & 0 & 1 & 0 & 1 & 0 & 1 & 0 & 0 \\
7 & 1 & 0 & 0 & 0 & 0 & 1 & 1 & 0 & 0 \\
8 & 0 & 1 & 0 & 0 & 0 & 1 & 1 & 0 & 0 \\
9 & 0 & 0 & 1 & 0 & 0 & 1 & 1 & 0 & 0 \\
10 & 1 & 0 & 0 & 1 & 0 & 0 & 0 & 1 & 0 \\
11 & 0 & 1 & 0 & 1 & 0 & 0 & 0 & 1 & 0 \\
12 & 0 & 0 & 1 & 1 & 0 & 0 & 0 & 1 & 0 \\
13 & 1 & 0 & 0 & 0 & 1 & 0 & 0 & 1 & 0 \\
14 & 0 & 1 & 0 & 0 & 1 & 0 & 0 & 1 & 0 \\
15 & 0 & 0 & 1 & 0 & 1 & 0 & 0 & 1 & 0 \\
16 & 1 & 0 & 0 & 0 & 0 & 1 & 0 & 1 & 0 \\
17 & 0 & 1 & 0 & 0 & 0 & 1 & 0 & 1 & 0 \\
18 & 0 & 0 & 1 & 0 & 0 & 1 & 0 & 1 & 0 \\
19 & 1 & 0 & 0 & 1 & 0 & 0 & 0 & 0 & 1 \\
20 & 0 & 1 & 0 & 1 & 0 & 0 & 0 & 0 & 1 \\
21 & 0 & 0 & 1 & 1 & 0 & 0 & 0 & 0 & 1 \\
22 & 1 & 0 & 0 & 0 & 1 & 0 & 0 & 0 & 1 \\
23 & 0 & 1 & 0 & 0 & 1 & 0 & 0 & 0 & 1 \\
24 & 0 & 0 & 1 & 0 & 1 & 0 & 0 & 0 & 1 \\
25 & 1 & 0 & 0 & 0 & 0 & 1 & 0 & 0 & 1 \\
26 & 0 & 1 & 0 & 0 & 0 & 1 & 0 & 0 & 1 \\
27 & 0 & 0 & 1 & 0 & 0 & 1 & 0 & 0 & 1 \\
\hline
\end{tabular}

$v_{n N}$ de manera sencilla aplicando la ley de las tensiones de Kirchhoff:

$$
v_{n N}=\frac{v_{a N}+v_{b N}+v_{c N}}{3}
$$

De esta manera, la tensión de fase efectiva está dada por:

$$
\begin{aligned}
& v_{a n}=v_{a N}-v_{n N} \\
& v_{b n}=v_{b N}-v_{n N} \\
& v_{c n}=v_{c N}-v_{n N}
\end{aligned}
$$




\section{B. Modelado del filtro de salida}

En la Fig. 1 es posible apreciar el filtro de salida (LC) en cada una de las fases del MC conectado entre el sistema de generación y la red en el punto de acoplamiento común (PCC) a través de un interruptor de bypass. Cada pierna del convertidor posee un inductor de inductancia $L_{f}$ y su correspondiente resistencia en serie $R_{f}$ y un capacitor de capacitancia $C_{f}$. Considerando como variables de estado para este sistema de segundo orden la corriente a través de inductor $i_{L}$ y la tensión del capacitor $v_{c}$ y suponiendo que los parámetros son iguales en las tres piernas, la dinámica del sistema en el plano $\alpha-\beta$ está dada por:

$$
L_{f} \frac{d i_{L \alpha \beta}}{d t}=v_{\alpha \beta}-v_{c \alpha \beta}-R_{f} i_{L \alpha \beta},
$$

donde $v_{\alpha \beta}$ corresponde a uno de los vectores válidos mostrados en la Tabla. I. Por otro lado, el comportamiento dinámico de la tensión a través del capacitor del filtro es definido como sigue:

$$
C_{f} \frac{d v_{c \alpha \beta}}{d t}=i_{L \alpha \beta}-i_{g \alpha \beta},
$$

De esta manera, la representación en el espacio de estados para el sistema está dada por:

$$
\frac{d \mathbf{x}}{d t}=\mathbf{A x}+\mathbf{B u}
$$

donde

$$
\begin{aligned}
& \mathbf{x}=\left[\begin{array}{c}
i_{L \alpha \beta} \\
v_{c \alpha \beta}
\end{array}\right], \quad \mathbf{A}=\left[\begin{array}{cc}
-\frac{R_{f}}{L_{f}} & -\frac{1}{L_{f}} \\
\frac{1}{C_{f}} & 0
\end{array}\right], \\
& \mathbf{u}=\left[\begin{array}{c}
v_{\alpha \beta} \\
i_{g \alpha \beta}
\end{array}\right] \text { and } \mathbf{B}=\left[\begin{array}{cc}
\frac{1}{L_{f}} & 0 \\
0 & -\frac{1}{C_{f}}
\end{array}\right],
\end{aligned}
$$

Las ecuaciones anteriores definen completamente el modelo continuo para el filtro $L C$ tomando como entradas por un lado la tensión de salida del MC $v_{\alpha \beta}$ y por el otro la corriente de salida inyectada a la carga $i_{g \alpha \beta}$.

\section{Modelo en tiempo discreto para el filtro LC}

El modelo discreto del sistema se obtiene como:

$$
\mathbf{x}(k+1)=\mathbf{A}_{d} \mathbf{x}(k)+\mathbf{B}_{d} \mathbf{u}(k),
$$

siendo $\mathbf{A}_{d}=e^{\mathbf{A} T_{s}}, \mathbf{B}_{d}=\int_{0}^{T_{s}} e^{\mathbf{A}\left(T_{s}-\tau\right)} \mathbf{B} d \tau$ y $T_{s}$ corresponde al tiempo de muestreo. Entonces, usando el modelo discreto para el sistema es posible predecir los estados de $v_{o}$ e $i_{L}$ como sigue:

$$
\begin{aligned}
& i_{L}(k+1)=a_{11} i_{L}(k)+a_{12} v_{c}(k)+b_{11} v(k)+B_{12} i_{g}(k), \\
& v_{c}(k+1)=a_{21} i_{L}(k)+a_{22} v_{c}(k)+b_{21} v(k)+b_{22} i_{g}(k),
\end{aligned}
$$

siendo:

$$
\mathbf{A}_{d}=\left[\begin{array}{ll}
a_{11} & a_{12} \\
a_{21} & a_{22}
\end{array}\right], \mathbf{B}_{d}=\left[\begin{array}{ll}
b_{11} & b_{12} \\
b_{21} & b_{22}
\end{array}\right],
$$

donde $k$ se refiere al instante de tiempo actual y $k+1$ indica el inicio del siguiente instante de muestreo. A partir de las ecuaciones anteriores es posible predecir los valores de la corriente en el inductor y la tensión de salida para utilizarlos en la implementación del control predictivo.

\section{Control Predictivo de Tensión}

El principio de funcionamiento básico del algoritmo es el siguiente. Al comienzo de cada instante de muestreo, se reciben nuevas medidas de $v_{c}, i_{L}$, e $i_{g}$. Estas mediciones definen el punto de partida desde el cual el algoritmo predice las trayectorias futuras de las variables de estado de acuerdo con (12) y (13), para cada vector de voltaje posible. Cada uno de esos valores predichos se evalúa luego con una función de costo $(\mathrm{CF})$ prediseñada, y se aplica un vector que corresponde al valor mínimo de CF al MC. La técnica propuesta en este apartado se basa en la presentada en [18]. La función de costo propuesta es la siguiente:

$$
g=\left(v_{o \alpha}^{*}-v_{o \alpha}\right)^{2}+\left(v_{o \beta}^{*}-v_{o \beta}\right)^{2}+\lambda_{d} g_{d e r},
$$

siendo $v_{o \alpha}^{*}$ y $v_{o \beta}^{*}$ las tensiones de referencia en el plano $\alpha-\beta$ y definiendo:

$g_{d e r}=\left(C_{f} \omega_{r e f} v_{o \beta}^{*}-i_{L \alpha}+i_{g \alpha}\right)^{2}+\left(C_{f} \omega_{r e f} v_{o \alpha}^{*}-i_{L \beta}+i_{g \beta}\right)^{2}$.

El término $g_{d e r}$ es introducido de manera a subsanar la incapacidad del esquema predictivo clásico de seguir la derivada de la tensión en el capacitor lo cual resulta en un alto nivel de distorsión armónica total (THD) creando de esta forma un regulador ideal que sigue tanto la tensión en el capacitor así como su derivada. El efecto del término derivativo es controlado por el factor de peso $\lambda_{d}$.

La estrategia se basa entonces en evaluar a cada instante de muestreo la función $g$ para cada uno de los vectores válidos y aplicar el vector que minimice la función de costo en el siguiente instante de muestreo.

\section{E. Generación de la tensión de referencia}

Como se ha mencionado anteriormente, el esquema propuesto se basa en la implementación de dos bucles de control, un lazo interno correspondiente al control predictivo de tensión y otro externo que genera la referencia de tensión para inyectar una corriente controlada a la red. En la Fig. 2 se muestra el lazo externo de control donde se calcula la diferencia entre la corriente de salida a la red $i_{g}$ y la corriente deseada $i_{g}^{*}$ para aplicarlos a un control PR al cual se le adiciona un control de prealimentación $v_{r e f}$ que se utiliza al arranque del sistema para sincronizar la tensión de salida de la etapa de conversión $v_{c}$ con la tensión de red $v_{g}$ de manera a conectarse en el PCC. Esta tensión de referencia generada es la entrada al bloque de control predictivo de tensión para calcular el vector óptimo a ser implementado. La tensión $v_{r e f}$ se genera a partir de la medición de la fase de la red $\theta$ obtenida a partir del PLL y la amplitud de la señal de la red. El valor de $i_{g}^{*}$ se determina a partir de la amplitud de corriente deseada y la fase deseada para la corriente de acuerdo a las potencias activa y reactiva. 


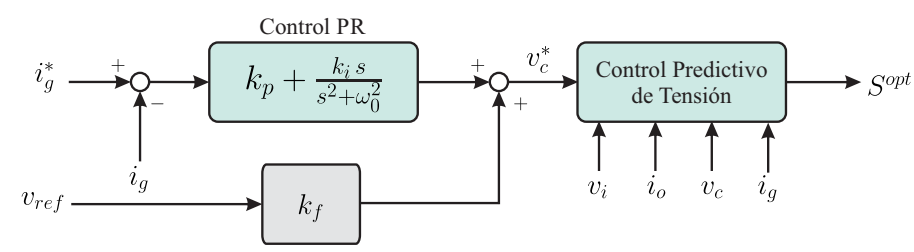

Fig. 2. Lazo externo de control con prealimentación.

\section{PRoceso de ARRANQUe Del SiStema y CONEXIÓN A LA RED}

El proceso de conexión a la red se realiza de la siguiente manera:

1) Se utiliza el control predictivo de tensión para obtener una tensión de salida del sistema igual a la tensión de la red, es decir, se sincroniza la tensión del capacitor con la red de manera a poder conectarlos al PCC.

2) Seguidamente, una vez que las tensiones son iguales, se utiliza el bypass para conectar los sistemas con la corriente de referencia de entrada igual a cero (esto es que ambas tensiones sigan siendo iguales).

3) Finalmente, se elimina la referencia generada por el sistema PLL y toda la tensión de referencia es proveída por el control PR, entonces es posible seleccionar la corriente a inyectar deseada.

Basado en el procedimiento descrito en el parágrafo anterior, es posible evaluar la propuesta en base a las distintas señales obtenidas.

\section{RESULTADOS OBTENIDOS PARA EL SISTEMA}

El esquema de control propuesto se valida a través de simulaciones realizadas utilizando la herramienta Simulink/Matlab. Los parámetros utilizados en la simulación se muestran en la Tabla II En la Fig. 3 se muestra la primera etapa del proceso para la interconexión del sistema con la red. Es posible observar como la tensión en el capacitor de salida paulatinamente sigue la tensión de la red, en aproximadamente

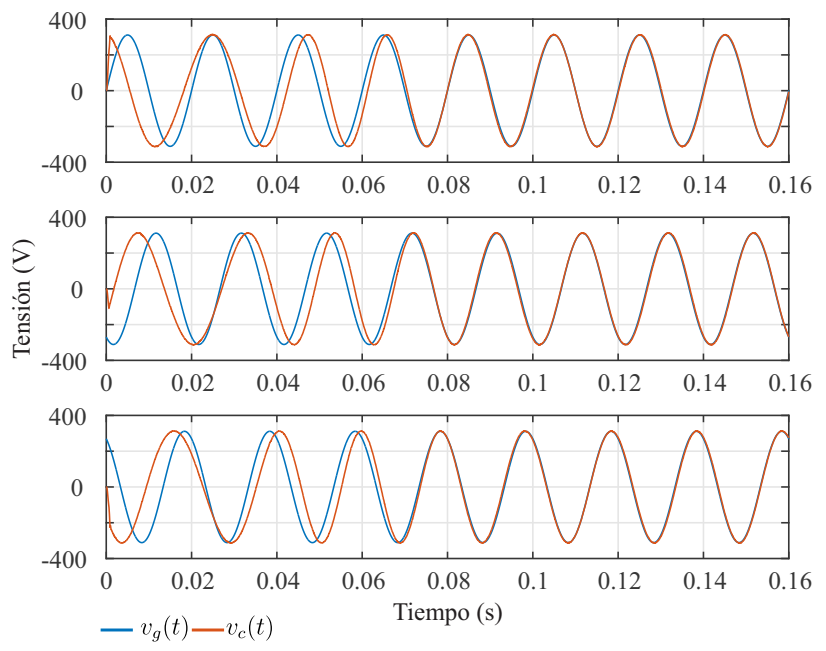

Fig. 3. Primera parte del proceso de conexión, tensiones sincronizándose.
TABLA II

PARÁMETROS UTILIZADOS EN EL ENTORNO DE SIMULACIÓN.

\begin{tabular}{lccc}
\hline & \multicolumn{3}{c}{ Parámetros de simulación } \\
\cline { 2 - 4 } Parámetro & Símbolo & Valor & Unidad \\
\hline \hline Tensión de la red & $v_{g}$ & 311 & $V_{p}$ \\
Frecuencia de la red & $f_{g}$ & 50 & $\mathrm{~Hz}$ \\
Resistencia del filtro & $R_{f}$ & 10 & $\mathrm{~m} \Omega$ \\
Inductancia del filtro & $L_{f}$ & 2.4 & $\mathrm{mH}$ \\
Capacitancia del filtro & $C_{f}$ & 24 & $\mu \mathrm{F}$ \\
Tiempo de muestreo & $T_{s}$ & 25 & $\mu s$ \\
Frecuencia de muestreo & $f_{s}$ & 40 & $\mathrm{kHz}$ \\
Peso del control derivativo & $\lambda_{d}$ & 0.2 & \\
Constante proporcional del PR & $k_{p}$ & 10 & \\
Constante integral del PR & $k_{i}$ & 1500 & \\
\hline
\end{tabular}

0,1 segundos ambas tensiones están sincronizadas y el sistema está listo para conectarse a la red.

Una vez que el sistema está sincronizado, el baypass cambia de posición y se inicia el proceso de inyección de corriente a la red. En este punto se debe asegurar que la corriente deseada esté configurada en cero amperes, lo que significa que las tensiones en los extremos de la inductancia de red son iguales. En la Fig. 4 se muestra el comportamiento de la corriente para una de las fases del sistema. Es posible observar aproximadamente a los 0.3 segundos un pequeño transitorio de corriente cuando el baypass conecta el sistema de generación con la carga. Luego de este punto, el valor de $k_{f}$ varía paulatinamente de 1 a 0 dejando sin efecto el control de prealimentación que inicialmente indicaba el valor de tensión de salida deseado para conectar los sistemas. aproximadamente a los 0.6 segundos se selecciona una corriente de referencia de 5 amperes y es posible observar como la corriente de la carga cambia a ese valor con una respuesta suficientemente rápida. A los 0.8 segundos se vuelve a cambiar la corriente de referencia a 10 amperes y se observa como la corriente inyectada cambia a ese valor.

Así también, es posible seleccionar la fase de la corriente a introducir a la red de manera a implementar esquemas de control de potencia activa y reactiva. En la Fig. 5 se muestra la respuesta del sistema para cambios de fase deseada en la corriente. En la mencionada figura, se presentan tanto la tensión de la red como la corriente inyectada. En la Fig. 5(a) se introduce un adelanto de 45 grados respecto a $v_{g}$, en la Fig. 5(b) se muestra un cambio en la fase de la corriente de

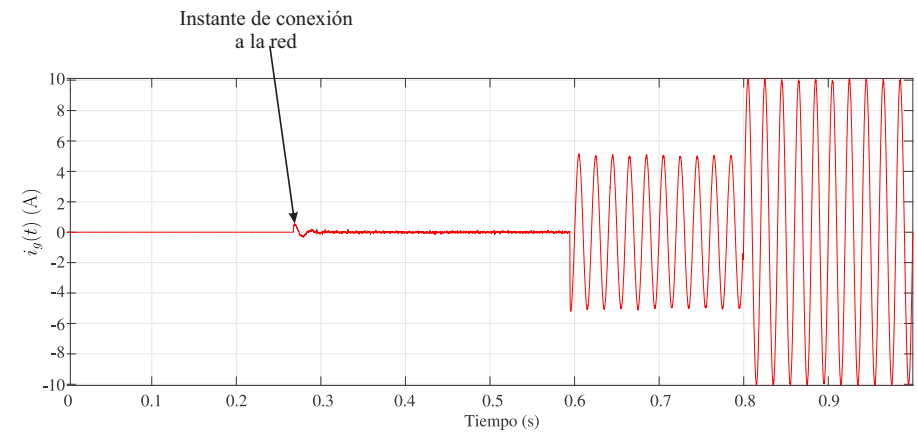

Fig. 4. Corriente inyectada a la red. 
(b)

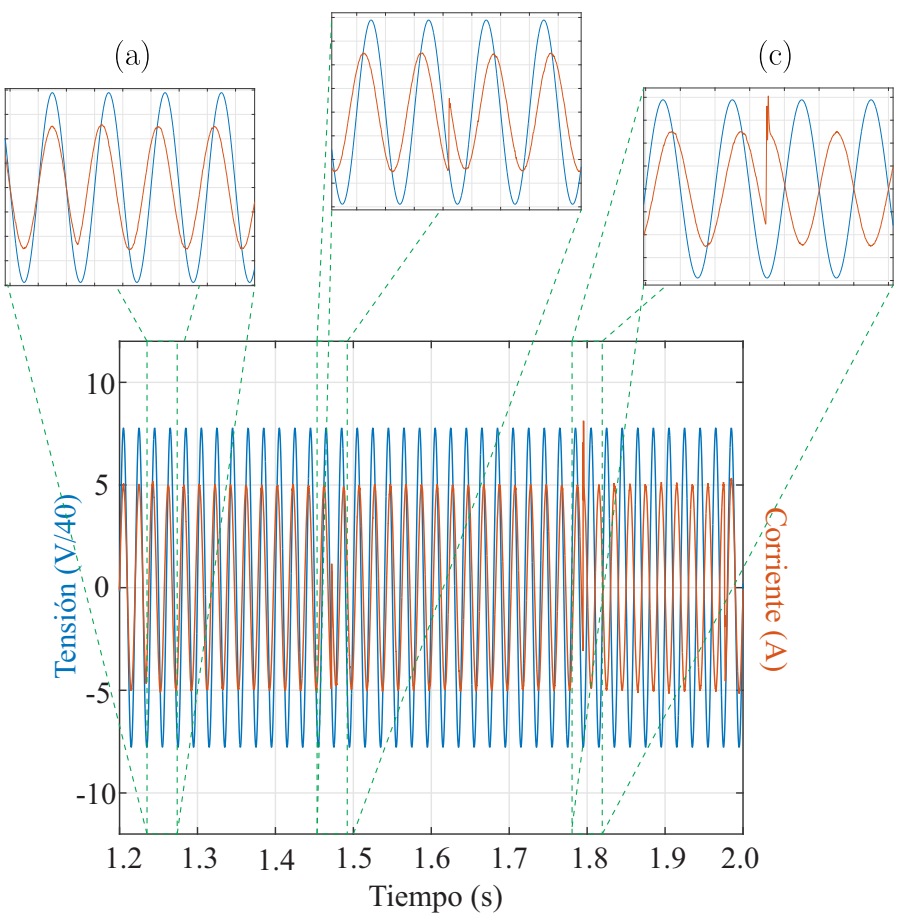

Fig. 5. Respuesta del sistema para cambios de fase.

45 grados de adelanto a 45 grados de atraso respecto a $v_{g} \mathrm{y}$ finalmente, en la Fig. 5(c) se muestra un cambio a un desfasaje de 180 grados entre la tensión $v_{g}$ y la corriente $i_{g}$.

En cuanto a la distorsión armónica total (THD) es posible observar que el nivel es de alrededor de $0.95 \%$, de hecho el promedio para cada una de las fases es similar al mostrado para la fase de la Fig. 6. Es posible apreciar entonces que el esquema cumple ampliamente con los estándares internacionales para sistemas interconectados a la red.

\section{CONCLUSiOnes}

El esquema propuesto en el presente trabajo ha mostrado un buen desempeño tanto en el arranque del sistema cuando se deben sincronizar las tensiones del sistema de generación y la red para conectarlos al punto de acoplamiento común como tras la activación del lazo externo para control de corriente inyectada a la red. El proceso de conexión del sistema
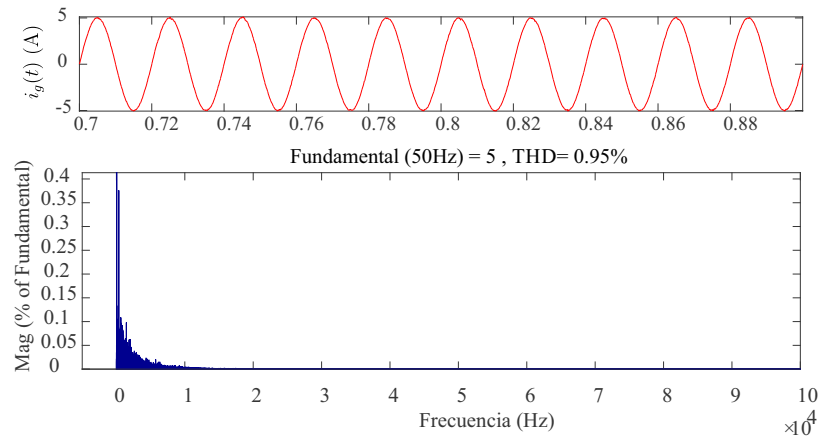

Fig. 6. Espectro de frecuencias de la corriente inyectada a la red. con la red ha sido efectivo y el control tanto de amplitud como de fase de corriente inyectada presenta un seguimiento correcto con alta calidad en las señales de salida cumpliendo ampliamente con los estándares internacionales. La propuesta constituye entonces una aplicación plausible para su posterior implementación experimental en sistemas de generación a partir de fuentes $\mathrm{AC}$ en contextos de generación distribuida.

\section{REFERENCIAS}

[1] F. Blaabjerg and K. Ma, "Wind energy systems," Proceedings of the IEEE, vol. PP, no. 99, pp. 1-16, 2017.

[2] V. Yaramasu, B. Wu, P. C. Sen, S. Kouro, and M. Narimani, "Highpower wind energy conversion systems: State-of-the-art and emerging technologies," Proceedings of the IEEE, vol. 103, no. 5, pp. 740-788, May 2015.

[3] S. Toledo, M. Rivera, and J. L. Elizondo, "Overview of wind energy conversion systems development, technologies and power electronics research trends," in 2016 IEEE International Conference on Automatica (ICA-ACCA), Oct 2016, pp. 1-6.

[4] V. P. K. Kunisetti, R. E. K. Meesala, and V. K. Thippiripati, "Improvised predictive torque control strategy for an open end winding induction motor drive fed with four-level inversion using normalised weighted sum model," IET Power Electronics, vol. 11, no. 5, pp. 808-816, 2018.

[5] J. Falck, G. Buticchi, and M. Liserre, "Thermal stress based model predictive control of electric drives," IEEE Transactions on Industry Applications, vol. 54, no. 2, pp. 1513-1522, March 2018.

[6] K. V. P. Kumar and T. V. Kumar, "Predictive torque control of open-end winding induction motor drive fed with multilevel inversion using two two-level inverters," IET Electric Power Applications, vol. 12, no. 1, pp. $54-62,2018$.

[7] K. M. R. Eswar, K. V. P. Kumar, and T. V. Kumar, "Modified predictive torque and flux control for open end winding induction motor drive based on ranking method," IET Electric Power Applications, vol. 12, no. 4, pp. 463-473, 2018.

[8] P. Wheeler, M. Rivera, and S. Toledo, "An indirect model predictive current control for a direct matrix converter with instantaneous reactive power minimization," in 2017 IEEE Southern Power Electronics Conference (SPEC), Dec 2017, pp. 1-6.

[9] F. Zhang, Y. Du, W. Liu, and P. Li, "Model predictive power control for cooperative vehicle safety systems," IEEE Access, vol. 6, pp. 47974810, 2018.

[10] M. Novak, U. M. Nyman, T. Dragicevic, and F. Blaabjerg, "Analytical design and performance validation of finite set mpc regulated power converters," IEEE Transactions on Industrial Electronics, pp. 1-1, 2018.

[11] T. Dragicevic, M. Alhasheem, M. Lu, and F. Blaabjerg, "Improved model predictive control for high voltage quality in microgrid applications," in 2017 IEEE Energy Conversion Congress and Exposition (ECCE), Oct 2017, pp. 4475-4480.

[12] S. Toledo, M. Rivera, R. Gregor, J. Rodas, and L. Comparatore, "Predictive current control with reactive power minimization in six-phase wind energy generator using multi-modular direct matrix converter," in 2016 IEEE ANDESCON, Oct 2016, pp. 1-4.

[13] M. Rivera, P. Wheeler, A. Olloqui, and D. A. Khaburi, "A review of predictive control techniques for matrix converters - Part I," in 2016 7th Power Electronics and Drive Systems Technologies Conference (PEDSTC), Feb 2016, pp. 582-588.

[14] _ - "A review of predictive control techniques for matrix converter Part II," in 2016 7th Power Electronics and Drive Systems Technologies Conference (PEDSTC), Feb 2016, pp. 589-595.

[15] S. Kouro, M. A. Perez, J. Rodriguez, A. M. Llor, and H. A. Young, "Model predictive control: Mpc's role in the evolution of power electronics," IEEE Industrial Electronics Magazine, vol. 9, no. 4, pp. 8-21, Dec 2015

[16] J. Falck, M. Andresen, and M. Liserre, "Thermal-based finite control set model predictive control for igbt power electronic converters," in 2016 IEEE Energy Conversion Congress and Exposition (ECCE), Sept 2016, pp. 1-7.

[17] H. Gao, B. Wu, D. . Xu, M. Pande, and R. P. Aguilera, "Common-modevoltage-reduced model-predictive control scheme for current-sourceconverter-fed induction motor drives," IEEE Transactions on Power Electronics, vol. 32, no. 6, pp. 4891-4904, Jun 2017.

[18] T. Dragičević, "Model predictive control of power converters for robust and fast operation of ac microgrids," IEEE Transactions on Power Electronics, vol. 33, no. 7, pp. 6304-6317, 2018. 